\title{
AMELIORATION DES CONNAISSANCES SUR LES REJETS PAR CLAPAGE DE TYPE VASE
}

\author{
Roland Boutin \\ Chef de la division études au service technique des travaux \\ immobiliers et maritimes, 15 rue de Laborde, 00309 ARMEES
}

Résumé

Les matériaux dragués régulièrement dans nos ports pour en garantir l'accessibilité sont en grande partie constitués de sédiments fins cohésifs (vases). Leur rejet en mer s'impose le plus souvent.

D'un point de vue théorique, l'état actuel des connaissances sur le comportement de ces matériaux lors de leur rejet en mer soulève encore de nombreuses questions. Des expériences en canal de laboratoire ont été réalisées afin d'étudier leur comportement à court terme. Différents résultats sont présentés. Un comportement de type "liqueur lourde" est notamment décrit. Il est également montré que le taux de dépôt dépend essentiellement de la rigidité initiale du rejet.

Deux modèles de simulation numérique ont été étudiés. L'analyse montre qu'ils doivent être utilisés avec circonspection pour des rejets de vases sur un site de faible profondeur soumis à des courants marins.

\section{Abstract}

Regurlarly dredged materials in harbour waters, in order to maintain accessibility, are mainly composed of cohesive mud. Usually, they have to be rejected into the sea.

On a theoritical point of view, the behavior of this kind of rejects still remains unclear, and subjected to many questions. Experiments are carried out in physical modelling flumes to study their behavior on a short time scale. Different results are presented here, and especially a "heavy liquor" one will be detailed. We point out that the deposition rate mainly depends on the initial yield value of the rejected material.

Two models of numerical simulations are studied. Their analysis show they have to be used with care for mud rejects in shallow waters submitted to marine currents.

\section{Introduction}

En France, 40 à 50 millions de mètres cubes de matériaux sont dragués chaque année dans les ports autonomes et les principaux ports d'intérêt national au titre des dragages d'entretien ${ }^{1}$. La présence de sédiments fins (vases) dans les

' travaux servant à maintenir ou améliorer les caractéristiques des chenaux d'accès et les cotes de fond des bassins portuaires afin de garantir leur accessibilité dans de bonnes conditions de sécurité. 
matériaux dragués rend leur réemploi ou valorisation difficile. Leur rejet en mer s'impose le plus souvent. Les phénomènes physiques qui gouvernent l'évolution à court terme d'un tel rejet recouvrent cependant une problématique complexe.

L'étude présentée ci-après cherche à améliorer les connaissances sur l'un des aspects de la problématique : le comportement à court terme des rejets par clapage $^{2}$ des produits de dragage d'entretien de type vase. Les résultats présentés sont issus de la thèse de Boutin (1999).

Après un point sur l'état actuel des connaissances, les principaux résultats d'expériences en laboratoire sont donnés. L'analyse de deux modélisations actuellement disponibles sera ensuite abordée. Quelques recommandations seront alors proposées.

\section{Etat actuel des connaissances}

Plusieurs auteurs ont décrit le comportement de matériaux rejetés en eaux libres (mer, lac, estuaire) par clapage (Bokuniewicz et al, 1978 ; Brandsma et Divoky, 1976 ; Gordon, 1974 ; Koh et Chang, 1973 ; Krishnappan ,1975; Tavolaro, 1984 ; Bokuniewicz et Gordon, 1980 ; Truitt, 1986 ; Tola et al, 1987). Ils fondent leurs analyses sur l'observation de différentes opérations en nature.

Bokuniewicz et al (1978) indiquent qu'un tel rejet pourra àvoir la consistance d'un fluide dense ou celle d'un ensemble d'éléments compacts pour des matériaux présentant une cohésion importante (galets de vase). Le mélange des deux est également possible. Tous les auteurs ne font pas cette distinction.

Ces matériaux chutent en masse dans la colonne d'eau sous l'action de leur flottabilité négative. La mixture est "le moteur" des phénomènes. Cette analyse est confirmée par Gordon (1974). Pour un rejet de silt et d'argile, il montre que la vitesse de chute du nuage (valeur moyenne de l'ordre de $40 \mathrm{~cm} . \mathrm{s}^{-1}$ ) est nettement supérieure à la vitesse de chute des particules individuelles estimée à $0,5 \mathrm{~cm} \cdot \mathrm{s}^{-1}$.

Dans l'approche de Bokuniewicz et al (1978), les galets de vase atteignent rapidement une vitesse limite qui dépend de leur taille. Les auteurs évoquent des vitesses de $1,53 \mathrm{~m} . \mathrm{s}^{-1}$ pour des galets ayant une taille de 13 centimètres. Ils incorporent peu d'eau mais subissent l'action des courants de la zone de rejet qui a tendance à déplacer le point d'impact au fond. Bokuniewicz et Gordon (1980) pensent qu'ils acquièrent la vitesse de déplacement latéral égale à celle du milieu récepteur. Si leur cohésion est suffisamment grande au regard de la vitesse d'impact, ils restent en place et participent à la formation d'un cône de dépôt lorsqu'ils touchent le fond. Dans le cas contraire, ils se disloquent et participent à la formation d'un fluide dense sur le fond.

Plusieurs auteurs indiquent que les rejets ayant une consistance fluide incorporent beaucoup d'eau ambiante au cours de la descente notamment du fait de la turbulence qui se développe à l'interface du jet dense avec le milieu récepteur. Cette observation est nuancée par Yauchi et Katoh (1992).

\footnotetext{
${ }^{2}$ vidange dans une zone d'immersion de l'ensemble des produits piégés dans le puits d'une drague.
} 
Pour ce qui est de la vitesse de chute, Bokuniewicz et al (1978) indiquent qu'elle est pratiquement constante lorsqu'il n'y a pas d'important gradient de densité dans le milieu récepteur. Cette vitesse semble principalement influencée par la vitesse initiale d'injection. Gordon (1974) mentionne au contraire que cette vitesse décroît avec la profondeur. Suivant les sites, les outils de dragage et les matériaux rejetés, des valeurs pouvant aller jusqu'à $2,8 \mathrm{~m} . \mathrm{s}^{-1}$ sont évoquées.

Une fraction très peu dense du rejet peut également être soustraite au jet fluide et à sa dynamique. Il y a alors formation d'un nuage de matériaux faiblement concentré qui s'échappe de la zone du rejet sous l'effet de l'hydrodynamisme du site.

Pour la problématique française de clapage par faible profondeur, le rejet rencontre le fond dans une phase dite "d'impact dynamique au fond". Il y a alors extension sur le fond sous forme de courants de densité qui seront éventuellement augmentés de ceux générés par la dislocation des galets de vase évoqués précédemment. Gordon (1974), Truitt (1986), Bokuniewicz et al (1980) indiquent que les matériaux se déplacent sur le fond en restant confinés dans une couche dont l'épaisseur représente environ 15 à $20 \%$ de la hauteưr d'eau au site de dépôt. Cette extension se poursuit jusqu'à ce que les énergies potentielles et cinétiques du jet à l'impact soient dissipées. Lorsque la vitesse de déplacement de la mixture est suffisamment faible, les matériaux la constituant décantent. Au point d'impact, il peut également y avoir érosion des matériaux du fond qui sont alors intégrés au flux de matière en déplacement. La forme et l'importance de l'étalement dépendront en particulier de la vitesse d'impact, de la densité de la mixture et de ses propriétés rhéologiques au moment de l'impact, du courant ambiant au fond, de la nature et de la topographie des fonds. Si l'énergie du jet à l'impact est trop faible, il y aura très peu d'extension latérale et pas d'érosion des matériaux du fond. Un dépôt en forme de cône se formera.

Malherbe (1989) analyse d'une toute autre façon le comportement des matériaux rejetés par clapage. Il confirme l'existence de phases de chute, d'impact au fond et de propagation sur le fond, mais évoque la ségrégation des matériaux pour expliquer leur comportement. L'observation de clapages de matériaux sablovaseux du port de Zeebruge dragué par une drague aspiratrice en marche, montre qu'après une phase de chute sous forme de courant de densité verticale ${ }^{3}$, les matériaux grossiers non cohésifs restent à proximité du point d'impact alors que les vases se propagent sur le fond sur une distance de 2 kilomètres en 2 heures. La forme du dépôt est influencée par les courants de marée existant dans la zone. Les mêmes types d'observations sont faites lors de suivis expérimentaux au Kannick, à Octeville ou au cap d'Antifer (Caillot et Sauzay, 1973, Hoslin et al, 1989, Tola, 1984).

Une fois les phénomènes décrits précédemment achevés, les sédiments pourront rester sur le fond où ils subiront des phénomènes de tassement et de consolidation. Ils pourront également être repris par les courants ambiants. Dans ce cas, les matériaux du rejet n'ont plus de dynamique propre. Des phénomènes de

\footnotetext{
${ }^{3}$ l'auteur indique que 95 à $99 \%$ des matériaux rejetés chutent au fond.
} 
convection sous l'effet des courants ou de diffusion sous l'effet de la turbulence ambiante sont alors observés. Les mécanismes en jeu sont identiques à ceux utilisés pour les modèles de sédimentologie côtière. Malherbe (1989) montre que ces phénomènes peuvent être très importants puisque dans le cas de Zeebruge, 70 à $80 \%$ des matériaux initialement déposés au fond disparaissent en 24 heures.

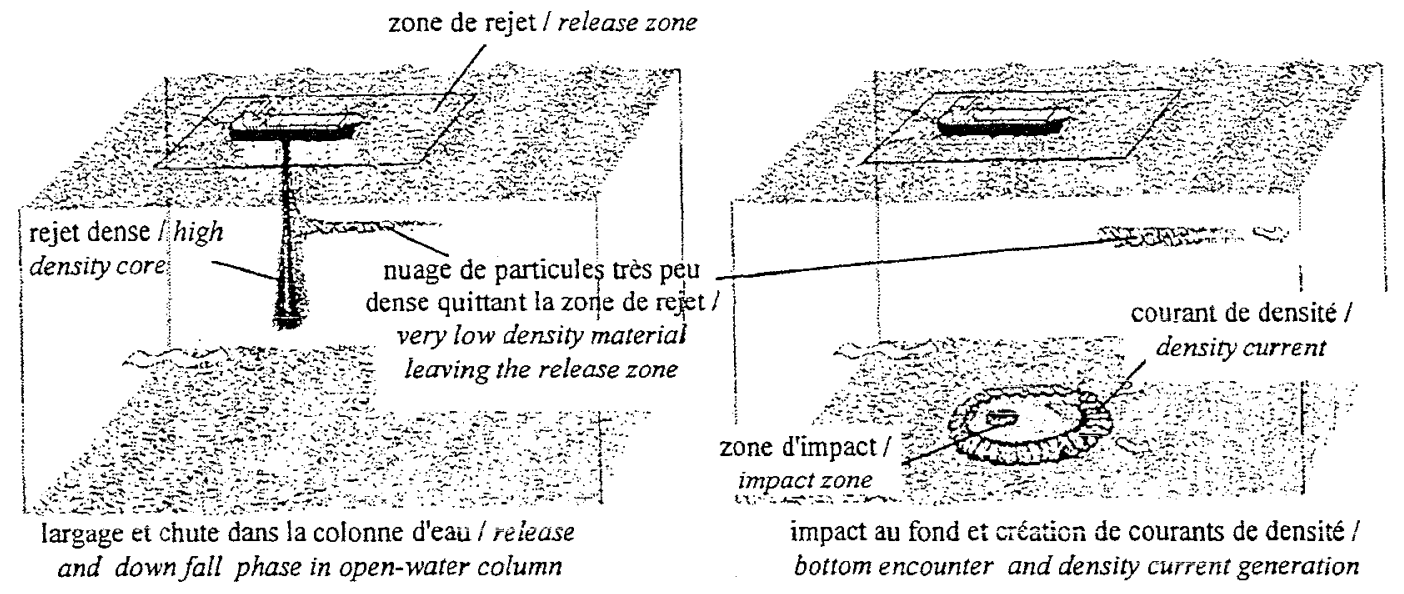

Figure 1 : Schématisation du comportement d'un rejet en eary libres avec impact au fond (adapté de Truitt, 1986 par Boutin, 1999)/transport processes during openwater disposal with bottom encounter (adapted from Truitt, 1986 by Boutin, 1999)

Les observations et interprétations qui viennent d'être rappelées montrent que s'il y a bien un consensus sur l'analyse qui est faite des phénomènes généraux (décomposition des phénomènes en quelques étapes bien identifiées, voir figure 1), des divergences apparaissent lorsqu'on affine davantage l'observation. Nous avons cherché à mieux comprendre les phénomènes mis en jeu par le biais d'études en laboratoire.

\section{Expériences en canal de laboratoire}

\subsection{Objectif et limitation}

Le domaine étudié fait intervenir de multiples facteurs (matériels, physiques, chimiques, dynamiques...). Le canal de laboratoire permet de contrôler les conditions d'essais. Pour le clapage, il a été possible d'étudier l'influence de la concentration en matière sèche de la mixture au moment du rejet ainsi que celle de la vitesse du courant de la veine liquide. Un modèle simplifié au $1 / 25^{\text {eme }}$ a été utilisé. Nous avons montré que la similitude avec les phénomènes en nature est correcte tant que les écoulements sont turbulents et que le rejet peut être considéré comme une suspension.

\subsection{Description des expériences réalisées}

Les expériences ont été réalisées dans un canal ${ }^{4}$ ayant une section utile de $1,5 \times 1,5 \mathrm{~m}^{2}$ et une longueur de $70 \mathrm{~m}$. Pour les essais de clapage la hauteur d'eau

${ }^{4}$ canal 5 du Centre de recherche d'électricité de France (EDF) à Chatou. 
était de $1 \mathrm{~m}$ (soit $25 \mathrm{~m}$ en nature) et le courant a varié suivant les expériences de 0 à $20 \mathrm{~cm} \cdot \mathrm{s}^{-1}$ ( 0 à $1 \mathrm{~m} \cdot \mathrm{s}^{-1}$ en nature). Un godet à ouverture par commande pneumatique assurait le rejet de la mixture de vase. Le sédiment utilisé pour les expériences était une vase naturelle prélevée dans le port du Havre, tamisée à $125 \mu \mathrm{m}$. La concentration initiale des rejets en matière sèche a varié de $180 \mathrm{~g} / \mathrm{l}$ à $420 \mathrm{~g} / \mathrm{l}$ en fonction des expériences. Pour les essais présentés ci-après, le volume

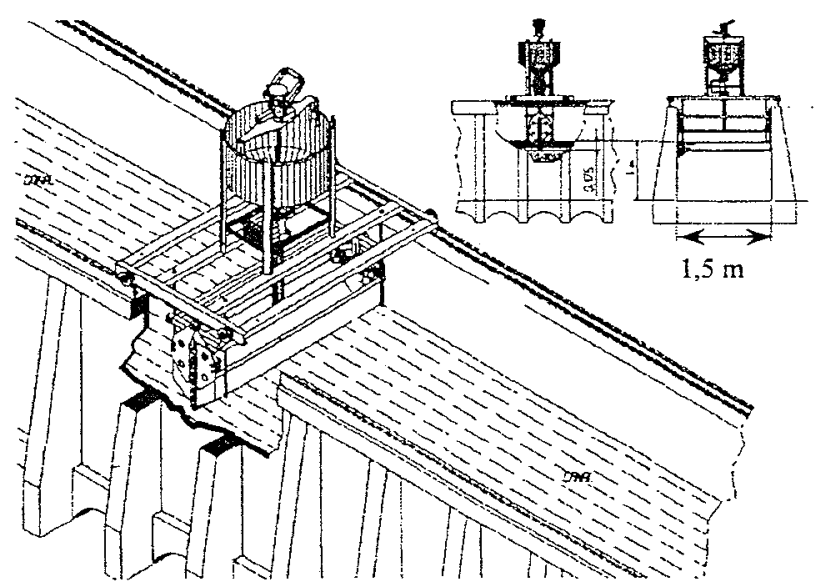

Figure 2 : dispositif de rejet / disposal equipment de matériaux introduit dans le canal est d'environ 44 litres. Différentes sections du canal ont été équipées de dispositifs de mesure afin de rendre compte de l'évolution du rejet dans le temps. La concentration en matière sèche a notamment été mesurée à l'aide d'une trentaine de sondes à scintillation excitées par la vase préalablement marquée à l'indium. Cette technique a permis de mesurer avec précision les répartitions des dépôts formés sur le fond du canal.

\subsection{Résultats}

Les moyens mis en œuvre et le nombre d'expériences réalisées permettent de mettre en évidence différents types de comportement physiques. Nous ne développerons ici que les résultats les plus marquants.

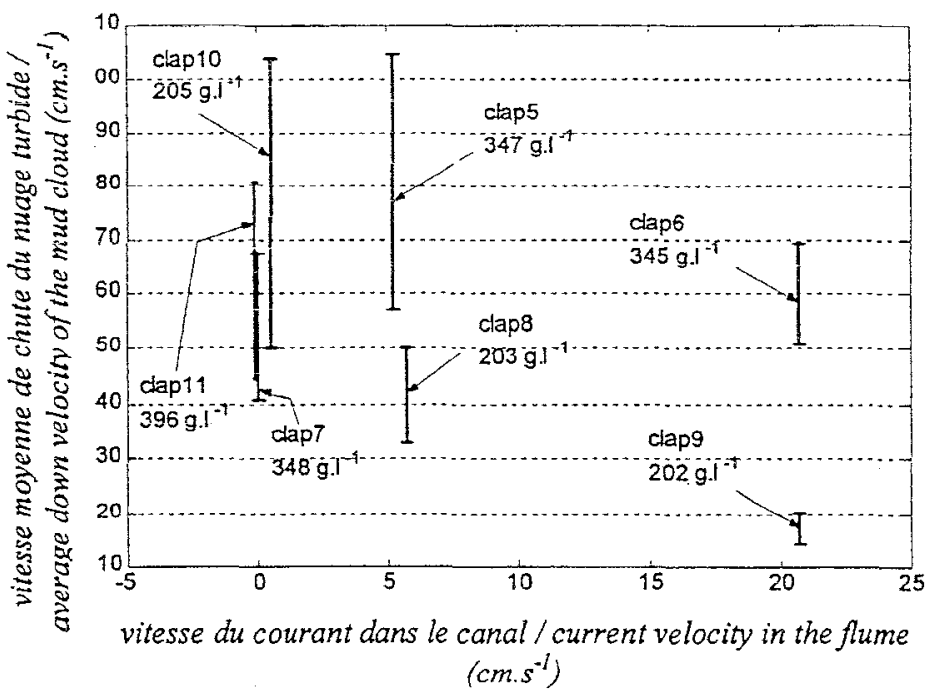

Figure 3 : vitesse de chute moyenne du nuage turbide pour différents essais avec rejet juste au-dessus de la surface libre / average down velocity of the mud cloud for different tests with reject just above the free surface
Les figures 3 et 4 illustrent les valeurs de vitesse moyenne de chute des nuages turbides obtenues pour les différents essais. La figure 3 concerne des essais avec le godet juste au dessus de la surface libre. La figure 4 concerne des essais avec le godet immergé de $17,5 \mathrm{~cm}$ dans l'eau. Un premier résultat s'impose : les vitesses de chute observées sont plus de 1000 fois supérieures 
aux vitesses de chute individuelles des particules fines qui constituent les nuages turbides. Le rejet se comporte comme une "liqueur lourde".

Pour les essais à concentration élevée, on peut observer que le rejet est constitué de 2 ou 3 nuages successifs. Les matériaux sont libérés par paquets à cause de l'action combinée de la forte rigidité initiale de la mixture dans le godet et de la réduction de l'effet de la pesanteur par l'immersion. Ce phénomène n'est pas observé lorsque le godet est placé au dessus de la surface libre.

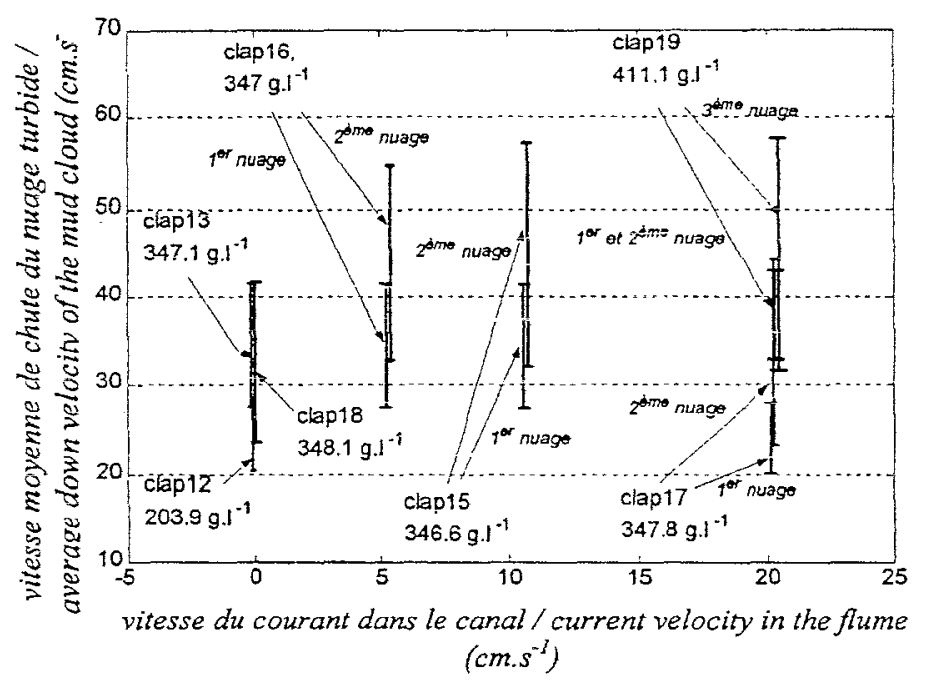

Figure 4 : vitesse de chute moyenne du nuage turbide pour différents essais avec rejet $17,5 \mathrm{~cm}$ sous la surface libre / average down velocity of the mud cloud for different tests with reject under $17,5 \mathrm{~cm}$ from the free surface

Pour les essais sans courant et godet au dessus de l'eau (voir figure 3), l'augmentation de la concentration initiale du rejet entraîne une réduction de la vitesse de chute. Ce résultat peut s'expliquer par une diminution de la vitesse initiale d'injection des matériaux du fait de l'augmentation de leur viscosité. Ce résultat n'est pas observé lorsque le godet est légèrement immergé (voir figure 4). On peut comprendre que dans ce cas la vitesse d'injection joue un rôle moins important que la flottabilité négative du nuage. Pour les essais avec courant, l'augmentation de la concentration initiale du rejet entraîne une augmentation de la vitesse de chute. L'augmentation de la vitesse du courant dans le canal tend au contraire à réduire la vitesse de chute des nuages turbides. Cette diminution est cependant assez faible lorsque le godet est immergé. On peut retenir que lorsque le godet est immergé, la flottabilité négative du nuage est une action dominante. Son effet est réduit avec l'augmentation de la vitesse d'écoulement de la veine d'dans le canal.

Une fois au fond, des courants turbides se propagent emportant avec eux une fraction des matériaux largués. Nous retrouvons là les observations générales rappelées au paragraphe 2 . La répartition des matériaux sur le fond est très sensible aux conditions initiales du rejet et en particulier à la concentration (voir figures 5 et 6 ). Pour les rejets avec le godet dans l'eau à concentration initiale élevée, la zone de dépôt est toujours peu étendue quelle que soit la vitesse dans le canal. Il ressort également que sans courant dans le canal, les matériaux rejetés restent dans le champ proche quelle que soit la concentration initiale du rejet ou la position du godet. 
Avec du courant dans le canal, les rejets présentant une rigidité initiale inférieure à 1 ou $2 \mathrm{~N} . \mathrm{m}^{-2}$ (environ $250 \mathrm{~g} . \mathrm{I}^{-1}$ pour la vase utilisée) sont rapidement emportés en dehors du champ proche. Le taux de dépôt est dans ce cas très sensible à la concentration initiale et à l'intensité du courant dans le canal. Lorsque la rigidité initiale est supérieure à 1 ou $2 \mathrm{~N}^{-\mathrm{m}^{-2}}$ plus de $80 \%$ des matériaux restent dans le champ proche quelle que soit la position du godet ou la vitesse dans le canal (voir figure 7).

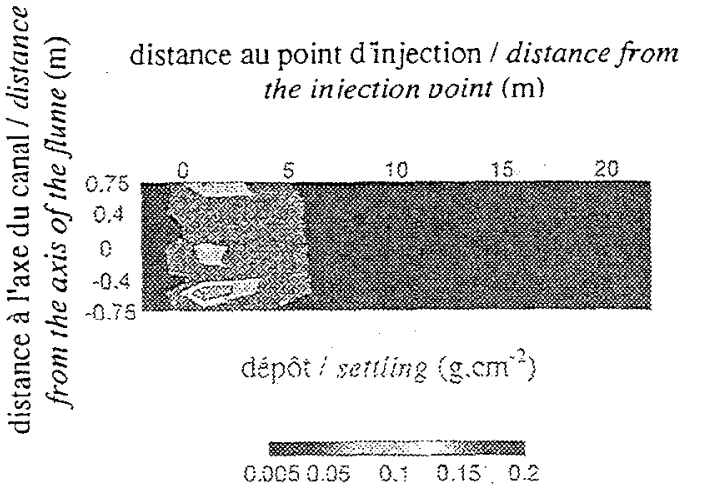

Figure 5 : cartographie du dépôt de l'essai clapp à $348 \mathrm{~g} . \mathrm{l}^{-1}$, vitesse du courant dans le canal :

$5,2 \mathrm{~cm} \cdot \mathrm{s}^{-1} /$ mapping of settling for test clap 5 at $348 \mathrm{~g} . \mathrm{l}-1$, current velocity in the flume : $5.2 \mathrm{~cm}^{-1} \mathrm{~s}^{-1}$

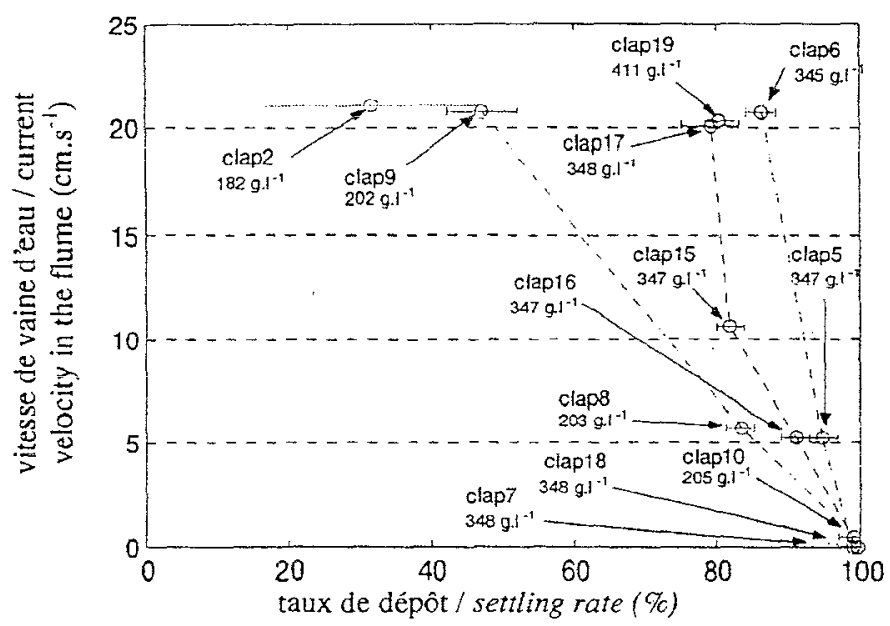

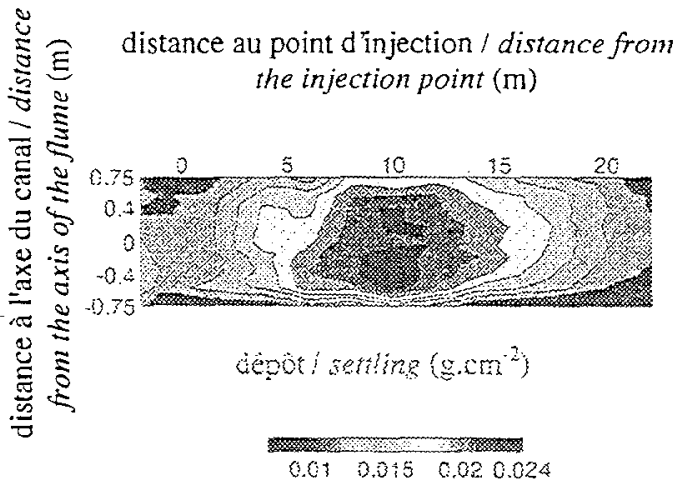

Figure 6 : cartogràphie du dépôt de l'essai clap8 à $203 \mathrm{~g} . \mathrm{l}^{-1}$, vitesse du courant dans le canal : $5,7 \mathrm{~cm} \cdot \mathrm{s}^{-1} /$ mapping of settling for test clap 5 at $203 \mathrm{~g} . \mathrm{l}-\mathrm{l}$, current velocity in the flume: $5.7 \mathrm{~cm}^{-1} \mathrm{~s}^{-1}$

Figure 7 : Taux de dépôt en fonction de la vitesse du courant dans le canal I settling rate in function of the current velocity in the flume

Les expériences réalisées ont également montré que lorsque le milieu récepteur est animé d'un courant, une dynamique complexe se met en place avec des phénomènes de dépôt et de reprise des matériaux.

Les différents résultats obtenus en canal nous amènent à penser que le comportement du rejet avec courant ne peut pas être déduit simplement du comportement du rejet sans courant. Comme nous allons le montrer dans le 
paragraphe suivant, cette analyse a des conséquences importantes sur la validité des modèles de simulation actuellement disponibles.

\section{Analyse de deux modèles de simulation}

Nous avons testé la modélisation développée par Krishnappan (1975) et celle du modèle STFATE de U.S. Army Corps of Engineers.

\subsection{Modèle de Krishnappan (1975) complétée par Drapeau et al (1992)}

Krishnappan (1975) propose d'évaluer la vitesse de chute et le rayon d'un rejet lorsque celui-ci atteint le fond de la zone où il a été largué. La démarche s'appuie sur une analyse dimensionnelle et des expériences réalisées en laboratoire. Le modèle peut être complété par les propositions de Drapeau et al (1992) en ce qui concerne l'estimation de la quantité de matériaux restant au fond.

La confrontation de certains résultats obtenus en canal avec ceux donnés par le modèle pour des conditions initiales comparables montre:

- que sans courant dans le canal, l'ordre de grandeur des vitesses de chute moyennes fournies par le modèle de Krishnappan n'est pas aberrant tant que le godet est hors de l'eau. Celui-ci est cependant largement surestimé lorsque le godet est immergé, ce qui traduit une nette sous-estimation de la durée de la phase de chute,

- que pour les essais avec courant dans le canal et godet dans l'eau, la sousestimation de la durée de chute est amplifiée,

- que la modélisation du taux de dépôt proposé n'est pas applicable pour les sédiments fins cohésifs utilisés.

Il s'en suit que la modélisation de Krishnappan (1975) complétée par Drapeau et al (1992) ne fournit pas de résultats très convaincants dans le cas de rejets de sédiments fins cohésifs (vases), notamment lorsque le milieu récepteur est en mouvement sous l'effet d'un courant.

\subsection{MIodèle STFATE}

La théorie du modele repose sur les travaux de Koh et Chang (1973) qui ont proposé une analyse et un modèle mathématique afin de simuler le comportement des rejets en mer. Durant la phase de chute, le nuage est supposé a priori hémisphérique. Brandsma et Divoky (1976) puis Johnson et al (1993 a et b) ont par la suite développé cette approche pour finalement aboutir au modèle STFATE.

La confrontation de certains résultats obtenus en canal avec ceux donnés par le modèle pour des conditions initiales comparables montre :

- que le taux de dépôt obtenu par simulation pour des essais sans courant dans le canal est très correct (différence inférieure à 10\%),

- que les vitesses moyennes de chute sont également bien simulées pour des essais sans courant dans le canal, 
- que pour l'essai clap19 (411 g. $\left..^{-1}, 20 \mathrm{~cm} \cdot \mathrm{s}^{-1}\right)$ avec courant, le taux de dépôt obtenu par simulation est très inférieur à celui mesuré en canal (environ 4 fois plus faible), la forme du dépôt est également très différente.

Il s'en suit que le modèle STFATE représente de façon acceptable le comportement d'un rejet de type vase dans un milieu récepteur au repos. Ce modèle ne semble cependant pas en mesure de représenter correctement le devenir d'un rejet de vase par faible profondeur dans une veine d'eau animée d'un courant.

\section{Conclusion}

Comme nous l'avons développé, seul un consensus général sur les grandes phases d'un rejet semble pouvoir être dégagé. Les expériences en canal montrent que la mixture chute dans un premier temps sous forme d'une liqueur lourde ayant un comportement très particulier (vitesse de chute plus de 1000 fois supérieures aux vitesses individuelles des particules qui la constituent). Les phénomènes se développent ensuite au plus près du fond sous forme de courant de densité. Les possibilités de transport de cette mixture seront très dépendante de sa rigidité initiale.

Nos tests sur deux modèles de simulation actuellement disponibles montrent qu'ils doivent être utilisés avec circonspection pour des rejets de vase par clapage et par faible profondeur avec courant. Dans l'état actuel des connaissances sur ce type de rejet, noùs préconisons que les études de sites de dépôts soient étayées par des mesures de terrain avec des rejets expérimentaux suffisamment détaillés.

\section{Remerciements}

Les expériences réalisées ont principalement été financées par la "mission pour le développement de l'innovation participative" et le "fond d'intervention pour l'environnement" (FIE) du Ministère de la Défense, par le groupe d'étude et d'observation sur le dragage et l'environnement (GEODE) et par le centre d'études techniques maritimes et fluviales (CETMEF). Le service des travaux maritimes et notamment le service technique des travaux immobiliers et maritimes (STTIM) ont également apporté une très large contribution aux différentes phases de l'étude.

\section{Référence}

Bokuniewicz H.J., Gebert J, Gordon R.B., Higgins J.L., Kaminsky P, Pilbeam C.C., Reed M, Tuttle C. (1978) - "Field study of the mechannics of the placement of dredged material at open-water disposal sites", New Haven, Connecticut (USA), Yale University pour l'USAEWES. 318 p. TR D-78-7.

Bokuniewicz H.J., Gordon R.B. (1980) - "Deposition of dredged sediment at open watersites", Estuarine and coastal marine science, 1980, 10, pp 289-303. 
Boutin R. (1999) - "Amélioration des connaissances sur le comportement des rejets en mer de produits de dragage de type vase". Thèse de doctorat en génie civil, INSA de Lyon, 527 p.

Brandsma M.G., Divoky D.J. (1976) - "Development of models for prediction of short-term fate of dredged material discharged in the estuarine environment", Passadena, California, Tetra Tech Inc. for USAEWES. 133 p., Contract report D76-5.

Caillot A, Sauzay G. (1973) - "Etude du rejet des produits de dragage au large d'Octeville", Saclay: CEA/TAAR, 40p + annexe, Rapport TAAR/ARS/73-21.

Drapeau G, Lavallée D, Dumais J.F., Walsh G. (1992) - "Dispersion model of dredged spoil dumped in coastal waters", Coastal engineering, pp 3054-3067.

Gordon R.B. (1974) - "Dispersion of dredged spoil dumped in near-shore waters", Estuarine and coastal marine science, 1974, 2, pp 349-358.

Hoslin R, Caillot A, Brisset P, Massias J, Poggi A, Sannie G. (1989) - "Etude sur la réduction des dragages d'entretien - Expérimentation à l'aide de traceurs radioactifs", Gif-sur-Yvette, CEA/DAMRI pour P.A. de Rouen. Rapport ORIS/SAR/S/89-110/H.55.

Johnson, B.H., Mc Comas, D.N., Mc Van, D.C., Trawle, M.J., (1993a) "Development and verification of numerical models for predicting the initial fate of dredged material disposed in open water, Report 1, Physical model tests of dredged material disposal from a split-hull barge and multiple bin vessel", Vicksburg : USAEWES. 103 p. DRP-93-1.

Johnson, B.H., Fong, M.T., (1993b) : "Development and verification of numerical models for predicting the initial fate of dredged material disposed in open water. Report 2, Theoretical developments and verification results." Vicksburg : USAEWES. 103 p. DRP-93-1.

Koh R.C.Y., Chang Y.C. (1973) - "Mathematical model for dredged ocean disposal wastes", Washington : National environmental research center, corvalis for USEPA. 178 p. Report EPA-660/2-73-029.

Krishnappan B.G. (1975) - "Dispersion of granular material dumped in deep water", Burlington, Ontario : Environment Canada. 113 p. Scientific series n ${ }^{\circ} 55$.

Malherbe B (1989) - "Case study of dumping in open areas", Actes du séminaire international sur les aspects environnementaux liés aux activités de dragages, Nantes, 27 novembre-1 ${ }^{\text {er }}$ décembre 1989. Nantes : PANSN. pp 227-261.

Tavolaro J.F. (1984) - "A sediment budget study of clamshell dredging and ocean disposal activies in the New York Bight", Environ.Geaol.Water Sci., 1984, Vol 6, $\mathrm{n}^{\circ} 3 \mathrm{p}$ 133-140.

Tola F, Caillot A, Courtois G, Gourlez P, Hoslin R, Massias J, Quesney M, Sauzay G. (1987). - "Study of the evolution of dredged material discharges by means of radioactive tracers", International symposium of the use of isotope 
techniques in water resources development, Vienna, 30 march - 3 april 1987, pp 663 - 682. IAEA-SM-299/71.

Tola F. (1984) - "Etude du rejet de produits de dragage à l'aide de traceurs radioactifs. Transfert et dispersion des fines particules en suspension et évolution dans le temps des sédiments déposés sur le fond", Société hydrotechnique de France, XVIII ${ }^{\mathrm{e}}$ journée de l'hydraulique, Marseille, 11,12 et 13 sept 1984.8 p. Q1 rapport $\mathrm{n}^{\circ} 15$.

Truitt C.L. (1986) - "Environmental effect of dredging. Fate of dredged material during open-water disposal", Vicksburg : USAWES. 12 p. EEDP-01-2.

Yauchi E., Katoh K. (1992) - "Dispersion process and settlement pattern of mud dumped in oceans", Coastal engineering 1992, pp 2734-2744. 